\title{
SANEAMIENTO Y DETOXIFICACIÓN DE CARNE DEALPACA (Vicugna pacos) CON SARCOCISTOSIS MEDIANTE TRATAMIENTOS FÍSICOSY QUÍMICOS DE USO DOMÉSTICO
}

\author{
Sanitation and Detoxification of Alpaca Meat (Vicugna pacos) with \\ Sarcocystosis by Physical and Chemical Methods of Home Use
}

Cristhian Céspedes V. ${ }^{1}$, Miguel Vilca L. ${ }^{1,3}$, Daphne Ramos D. ${ }^{1}$, Rosa Sam T. ${ }^{2}$, Juan Lucas L. ${ }^{1}$

\section{Resumen}

\begin{abstract}
Se evaluaron métodos físicos y químicos de uso doméstico para el saneamiento y detoxificación de la carne de alpaca infectada con macroquistes de Sarcocystis aucheniae (Sa). Los tratamientos físicos fueron cocción y horneado a $80{ }^{\circ} \mathrm{C}$ por 5 minutos, y congelación por 10, 15 y 20 días; y los químicos fueron el marinado y salazón por 15 y 30 días, y marinado por 24 horas. Trozos de carne de alpaca con 180-200 macroquistes que recibieron uno de los tratamientos fueron administrados a perros, analizándose la presencia de ooquistes en heces durante 18 días. Asimismo, se prepararon lisados de macroquistes de $\mathrm{Sa}$ de las muestras tratadas y se inyectaron en conejos para determinar el efecto detoxificante de los tratamientos sobre el contenido proteínico de los macroquistes. Se logró sanear la carne infectada únicamente con los tratamientos físicos, en tanto que no se logró inhibir la actividad biológica tóxica de la proteína de los macroquistes.
\end{abstract}

Palabras clave: saneamiento, detoxificación, Sarcocystis aucheniae, macroquistes, carne de alpaca

\section{Abstract}

Physical and chemical methods of home use techniques for sanitation and detoxification of alpaca meat infected with macrocysts of Sarcocystis aucheniae were evaluated. The physical treatments were boiling and baking at $80^{\circ} \mathrm{C}$ for 5 minutes and freezing for 10, 15 and 20 days; and the chemical treatments were salting for 15 and 30 days, and marinated for 24 hours. Dogs were fed with bouts of treated alpaca meat containing 180-200 macrocysts and the presence of oocysts were analyzed in faeces for

\footnotetext{
${ }^{1}$ Laboratorio de Salud Pública y Salud Ambiental; ${ }^{2}$ Laboratorio de Microbiología y Parasitología Veterinaria, Facultad de Medicina Veterinaria, Universidad Nacional Mayor de San Marcos, Lima

${ }^{3}$ E-mail:mavl06@hotmail.com; mavilcal@unmsm.edu.pe
}

Recibido: 21 de mayo de 2012

Aceptado para publicación: 17 de febrero de 2013 
18 days. Besides, macrocysts lisates were injected to rabbits to evaluate the detoxifying effect of treatments on the protein content of the macrocysts. Only the physical treatments proved to be effective in sanitizing alpaca infected meat, whereas the toxin biological activity could not be altered.

Key words: sanitation, detoxification, Sarcocystis aucheniae, macrocyst, alpaca meat

\section{INTRODUCCIÓN}

La sarcocistosis o sarcosporidiosis es una enfermedad parasitaria limitante para la producción alpaquera, producida por protozoos del género Sarcocystis. Las especies $S$. aucheniae $(\mathrm{Sa})$ y S. lamacanis afectan a las alpacas (Guerrero et al., 1967; Leguía et al., 1989, 1990). Estos parásitos digenéticos requieren en su ciclo de vida de un hospedero definitivo carnívoro (cánidos) y un hospedero intermediario herbívoro (alpaca, llama, guanaco). En estos últimos se desarrolla la fase asexual (Fayer, 2004), dando origen a los esquizontes en el endotelio vascular y a los bradizoitos en quistes visibles en la musculatura esquelética y cardíaca (Leguía, 1991). La frecuencia en los camélidos sudamericanos (CSA) puede ser muy elevada, reportándose prevalencias de hasta 100\% (Castro, 1974; Mostajo, 1983).

La sarcocistosis en el hombre se presenta en dos síndromes. Uno similar a una intoxicación alimentaria y el otro como consecuencia de la fase intestinal de los sarcocistos (Piekarski et al., 1978; Moreno, 2003; Fayer, 2004). El consumo de carne infectada con Sa insuficientemente cocida produce transtornos gastroentéricos asociados a la sarcocistina, toxina presente en los quistes (Leguía, 1991; Subercaseaux, 1994; Leguía y Casas, 1999). Los casos zoonóticos, donde el hombre es el hospedero definitivo, están asociados al consumo de carne parasitada de bovino y cerdo por las especies $S$. hominis y $S$. suihominis, respectivamente (Piekarski et al., 1978; Fayer et al., 1979; Fayer, 2004). Asimismo, las precarias condiciones de vida, como las encontradas en las zonas altoandinas, predisponen una elevada presentación de sarcocistosis humana (Wilairatana et al., 1996; Clavel et al., 2001; Nichpanit et al., 2010).

Diversas investigaciones han sido desarrolladas para el control y prevención de la sarcocistosis a través de la interrupción del ciclo biológico del parásito y del saneamiento de la carne para consumo. En este último caso, se han aplicado tratamientos físicos y químicos con el objetivo de destruir al parásito e inactivar su toxina (Gorman et al., 1984; Euzéby, 1998; Leguía y Arévalo, 1990). En este contexto, el objetivo del presente estudio consistió en inactivar los quistes y la toxina del Sa en la carne de alpaca mediante tratamientos físicos o químicos de uso doméstico a fin de volver la carne como apta para su consumo.

\section{Materiales y Métodos}

\section{Diseño Experimental}

El estudio se realizó en las instalaciones de la Facultad de Medicina Veterinaria de la Universidad Nacional Mayor de San Marcos, Lima. Se utilizaron seis canales de alpacas de cinco años de edad y visiblemente infectadas con macroquistes de Sa. Los animales fueron beneficiados en el camal de la provincia de Huancavelica. Además, se usaron 21 conejos de raza California de 4 a 6 meses de edad, machos y hembras en buen estado de salud, para evaluar el efecto de la toxina de $\mathrm{Sa}$; y 20 cachorros caninos cruza- 


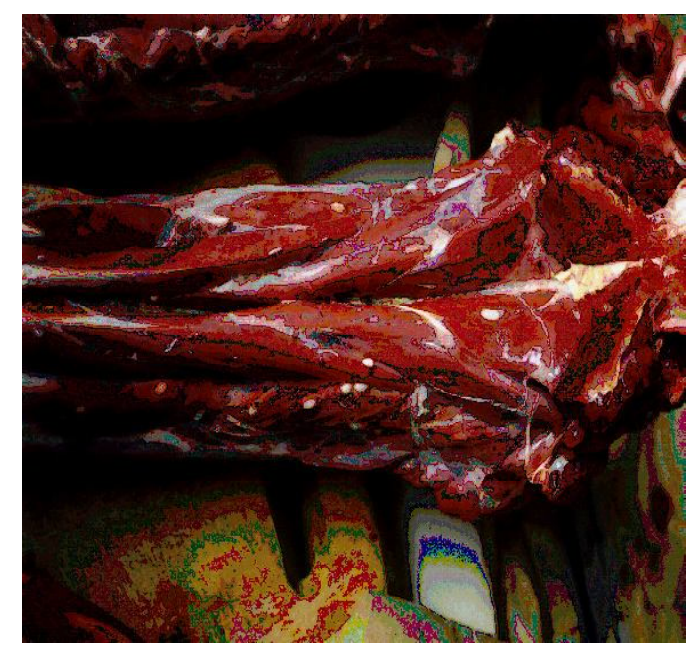

Figura 1. Carne de alpaca con macroquistes de Sarcocystis aucheniae

dos de 3 a 4 meses de edad, de ambos sexos en buen estado de salud, para evaluar la viabilidad de los quistes.

La carne de alpaca fue trozada en porciones de $200 \mathrm{~g}$, que contenían alrededor de 180 a 200 macroquistes cada una (Fig. 1). Tales porciones se distribuyeron en los siguientes tratamientos de tipo físico y químico de uso doméstico:

- T0: Control positivo (carne no tratada)

- T1: Cocción a $80^{\circ} \mathrm{C}$ por $5 \mathrm{~min}$

- T2: Horneado a $80^{\circ} \mathrm{C}$ por $5 \mathrm{~min}$

- T3: Congelación doméstica por 10 días (-18 ${ }^{\circ} \mathrm{C}$ como mínimo)

- T4: Congelación doméstica por 15 días (-18 ${ }^{\circ} \mathrm{C}$ como mínimo)

- T5: Congelación doméstica por 20 días (-18 ${ }^{\circ} \mathrm{C}$ como mínimo)

- T6: Salazón por 15 días (sal granulada, 16 $\mathrm{g}$ /porción de carne) y luego desalada en agua corriente por $24 \mathrm{~h}$

- T7: Salazón por 30 días (sal granulada, 16 g/porción de carne) y luego desalada en agua corriente por $24 \mathrm{~h}$

- T8: Marinado, $100 \mathrm{~g}$ de carne con $200 \mathrm{ml}$ de vinagre (5\% de ácido acético) y $5 \mathrm{~g}$ de sal, y en refrigeración por $24 \mathrm{~h}$

\section{Ensayo de Viabilidad de los Quistes}

Se asignaron dos perros por tratamiento, recibiendo cada uno una porción de carne tratada (200 g). Además, se tuvo un control negativo que fue alimentado con alimento balanceado comercial. Se realizaron exámenes coprológicos por el método de flotación con solución saturada de sal, antes de la ingestión de la carne y diariamente a partir del día 10 del consumo, para evaluar la presencia de esporoquistes en las heces. Los animales que no presentaron esporoquistes hasta el día 18 se consideraron negativos.

\section{Ensayo de Letalidad de la Toxina}

Preparación del inóculo. Se preparó un lisado de macroquistes de Sarcocystis (LMS) a partir de quistes libres de tejido muscular. Los quistes fueron triturados y tratados mediante ultrasonificación (Fisher Sonic Dismembrator 300) a 60 ciclos por 4 veces, con intervalos de $30 \mathrm{~s}$, para luego centrifugarlos a $12000 \mathrm{~g} / 30 \mathrm{~min}$. El sobrenadante del lisado centrifugado fue diluido en solución salina fosfatada $(\mathrm{pH} 7.2,0.15 \mathrm{M})$ para obtener un inóculo con un contenido mínimo de $100 \mu \mathrm{g}$ de proteína/ml.

Verificación de la letalidad del inóculo. Nueve conejos fueron inoculados con $1 \mathrm{ml}$ del LMS obtenido de las carnes de cada tratamiento (un conejo por tratamiento) para observar el efecto de la toxina. Los conejos restantes fueron inoculados con LMS tratados en autoclave según el protocolo siguiente:

- $121,100,80$ y $60^{\circ} \mathrm{C}$ por $10 \mathrm{~min}$ (un conejo por cada temperatura) para el control positivo

- $135{ }^{\circ} \mathrm{C}$ por 1 min para los inóculos con mayor poder tóxico (dos conejos)

- $100{ }^{\circ} \mathrm{C}$ por 10 min para el resto de inóculos (seis conejos, uno por tratamiento)

La cantidad del inóculo del LMS fue distinta en cada tratamiento debido al volumen obtenido a partir de su preparación. Se realizó la necropsia a los animales que mu- 
Cuadro 1. Periodo prepatente de Sarcocystis aucheniae en perros que consumieron carne de alpaca infectada con 180-200 macroquistes y sometida a tratamientos químicos

\begin{tabular}{llc}
\hline Grupo experimental & & Periodo prepatente (días) \\
\hline Control positivo & (T0) & $8-11$ \\
Salazón por 15 días & (T6) & $22-26$ \\
Salazón por 30 días & (T7) & $22-26$ \\
Marinado & (T8) & $15-18$ \\
\hline
\end{tabular}

rieron, tomándose muestras de hígado, riñón, bazo, corazón, pulmón, cerebro y cerebelo para el estudio histopatológico. Todas las muestras fueron fijadas en una solución de formol al 10\%, y embebidas en parafina, seccionadas y teñidas con hematoxilina y eosina.

\section{Resultados}

La viabilidad de los quistes de Sa fue afectada por los tratamientos físicos T1, T2, T3, T4 y T5 porque no se observó presencia de esporoquistes en las heces de los perros que consumieron esas carnes tratadas; sin embargo, los perros que consumieron la carne de los tratamientos T6, T7 y T8 eliminaron esporoquistes desde los 15 a los 26 días post-infección (Cuadro 1).

Ninguno de los perros presentó signos clínicos que puedan asociarse a la infección de Sarcocystis o al efecto de la toxina del parásito. Asimismo, ninguno de los ocho tratamientos inactivó la toxina ya que todos los conejos murieron entre 5 y $23 \mathrm{~h}$ post-inoculación del LMS. Los animales inoculados con LMS de carne no tratada (T0) murieron a las $12 \mathrm{~h}$.
Los signos clínicos en los conejos, caracterizados por postración, disnea, pupila contraída, hipertermia y ligera diarrea, se presentaron sin diferencias entre tratamientos, excepto por los inoculados con LMS del T2 y T8 donde se presentaron de forma más severa. Los inóculos del T2 y T8 fueron los más tóxicos, puesto que también causaron la mortalidad de los animales en el menor tiempo. Los resultados histopatológicos evidenciaron lesiones en todos los órganos evaluados, incluyendo congestión, necrosis, alteraciones vasculares y degeneración celular. Los órganos con más hallazgos patológicos fueron los pulmones, riñones, hígado y corazón.

El pulmón fue el órgano más afectado, mostrando una severa hiperemia, neumonitis y espasmo arterial y arteriolar, enfisema, colapso alveolar y hemorragia. Estas alteraciones están asociadas al efecto de la toxina sobre el endotelio vascular. En los riñones se observó el aumento moderado a severo del volumen de la estructura glomerular, con tumefacción glomerular, congestión y trastornos degenerativos del epitelio tubular. El hígado presentó una severa congestión de vasos portales y sinusoides hepáticos, así como la presencia de un proceso degenerativo agudo en hepatocitos, caracterizado por dege- 
neración turbia, hiperplasia de los conductos biliares y edema en el tejido conectivo de los espacios portales. En el miocardio se observaron trastornos circulatorios agudos, caracterizados por hiperemia, hemorragias y procesos degenerativos de fibras miocárdicas (desde degeneración hidrópica hasta degeneración de Zenker).

Los conejos que recibieron LMS previo pasaje por el autoclave a diferentes temperaturas no presentaron los efectos letales de la toxina.

\section{Discusión}

El saneamiento de la carne de alpaca infectada con Sa por los tratamientos físicos usados en este estudio (T1 a T5) permitió interrumpir el ciclo biológico del parásito, ya que los perros que ingirieron la carne tratada no eliminaron esporoquistes en las heces. Similares reportes se han documentado cuando la carne con macroquistes es cocinada a $60{ }^{\circ} \mathrm{C}$ o congelada a $-10{ }^{\circ} \mathrm{C}$ por 10 días (Gorman et al., 1984; Leguía y Arévalo, 1990). Asimismo, se dispone de reportes de saneamiento de la carne por calentamiento a $65-70{ }^{\circ} \mathrm{C}$ por 10 minutos y por congelación a $-20{ }^{\circ} \mathrm{C}$ por $10 \mathrm{~h}$ (Euzéby, 1998).

Granados et al. (2007) sanearon y detoxificaron con éxito la carne de llama con macroquistes de Sa a través del método químico de marinado, utilizando la misma concentración de sal y vinagre que en el presente trabajo; sin embargo, el marinado lo mantuvieron en refrigeración por $48 \mathrm{~h}$, esto, es el doble de tiempo que el utilizado en el T8, lo que podría indicar que el marinado sería más eficaz si se permite un mayor tiempo de refrigeración.

Otros tratamientos no evaluados en el presente estudio como la transformación de carne en charqui, chalona (Gorman et al., 1984; Leguía y Arévalo, 1990), curado seco, curado húmedo y ahumado (Granados et al., 2007) permitieron el saneamiento de la carne, además de la inactivación de la toxina, haciéndolas aptas para el consumo humano. Al parecer, la refrigeración no afectó la viabilidad de los quistes, confirmando reportes anteriores (Gorman et al., 1984; Leguía y Arévalo, 1990).

La presencia de esporoquistes en las heces de los perros control positivo en el presente estudio comprobó el alto nivel de infectividad de la carne con macroquistes de Sa. En general, la eliminación de los esporoquistes en las heces de los perros es variable (Leguía et al., 1989), con un periodo prepatente de 11 a 20 días y un periodo patente de 20 a 50 días. Las diferencias están asociadas al grado de infestación de la carne, viabilidad de los quistes, capacidad invasiva de los parásitos en la mucosa intestinal e inmunidad del hospedero. En el presente estudio, el periodo prepatente observado fue de 8 a 11 días.

La presencia de esporoquistes en las heces de los animales que consumieron carne sometida a tratamientos químicos (T6 a T8) fue señal de la falta de efectividad de los tratamientos sobre la viabilidad de los quistes; pese a que otros estudios habían demostrado que la salazón completa de carne infectada con Sarcocystis destruye al parásito (Rojas, 1990; Vilca, 1991). No obstante, la salazón (T6 y T7) tuvo un ligero efecto sobre el periodo prepatente del parásito, ya que la eliminación de esporoquistes ocurrió a partir de los 22 días post-infección.

Los tratamientos físicos interrumpieron el ciclo del parásito mas no la letalidad de la toxina, ya que todos los conejos murieron al ser inoculados con los lisados de macroquistes. Este inesperado resultado difiere de otros que reportaron que la cocción y congelación de carne infectada inactiva la toxina del parásito volviendo la carne apta para el consumo (Rojas, 1990; Subercaseaux, 1994; Granados et al., 2007). 
La liberación de la toxina del parásito estaría relacionada con la presentación, duración e intensidad del cuadro clínico. Los signos observados en este estudio fueron compatibles con otros estudios (Sam et al., 1998; Granados et al., 2007). La aplicación de tratamientos físicos mediante el incremento de la temperatura interna de la carne parasitada podría favorecer la liberación de la toxina, cuya estructura química se encontraría libre y penetraría con facilidad a las células de la mucosa intestinal y luego al torrente sanguíneo (Mansilla, 1993). La presentación de convulsiones en algunos casos está asociado a la actividad neurotóxica (Hiepe et al., 1981); en tanto que la hipertermia se atribuye a la toxina cuya presencia ocasiona la producción de pirógenos endógenos como mecanismo de defensa del organismo.

Las lesiones histopatológicas causadas por el LMS en los conejos fue similar a lo observado por Mansilla (1993), donde la muerte de los animales ocurrió en un lapso de 5 a 22 h post-inoculación, dependiendo de la dosis del inóculo. Los resultados histopatológicos demostraron que la toxina de Sa tiene gran poder tóxico, causando degeneración celular en pulmón, hígado, riñón y miocardio, siendo estos los órganos más afectados. Resultados similares fueron encontrados por Leguía et al. $(1989,1990)$ y Sam (1988).

El tratamiento de la carne en autoclave $\left(60\right.$ a $135^{\circ} \mathrm{C}$ ) afectó la letalidad de la toxina, de allí que se le podría considerar como una alternativa complementaria a evaluar para realizar el saneamiento de la carne.

\section{Conclusiones}

- Los tratamientos físicos de cocción, horneado y congelación de carne parasitada con Sarcocystis afectan la viabilidad del parásito, cortando el ciclo biológico y eliminando el riesgo de infección del hospedero definitivo.
- Los tratamientos químicos de salazón y marinado no afectaron la viabilidad de los quistes de Sarcocystis.

- Los tratamientos físicos y químicos no afectaron el efecto letal de la toxina de Sarcocystis.

- La aplicación del tratamiento térmico en autoclave demostró la detoxificación del lisado.

\section{Literatura Citada}

1. Castro J. 1974. Sarcocystis auchenia en llamas (Lama glama). Rev Inv Pec IVITA 3: 91-92.

2. Clavel A, Doiz O, Varea M, Morales S, Castillo FJ, Rubio MC, Gomez-Lus R. 2001. Molestias abdominales y heces blandas en consumidor habitual de carne de vacuno poco cocinada. Enferm Infec Microbiol Clin 19: 29-30.

3. Euzéby J. 1998. Les parasites des viandes: épidémiologie, physiopathologie, incidences zoonosiques. Paris: Tec \& Doc-Lavoisier. $402 \mathrm{p}$.

4. Fayer R. 2004. Sarcocystis in animal and human infections. Clin Microbiol Rev 17: 894-902.

5. Fayer R, Heydorn AO, Johnson AJ, Leek RG. 1979. Transmission of Sarcocystis suihominis from humans to swine to nonhuman primates (Pan troglodytes, Macaca mulatta, Macaca irus). Z Parasitenkd 59: 15-20.

6. Guerrero C, Hernández J, Alva J. 1967. Sarcocystis en alpacas. Rev Fac Med Vet 1(2): 69-76.

7. Gorman TR, Alcaíno HA, Muñoz H, Cunazza C.1984. Sarcocystis sp. in guanaco (Lama guanicoe) and effect of temperature on its viability. Vet Parasitol 15:95-101.

8. Granados L, Vilca M, Sam R. 2007. Saneamiento y detoxificación de carne de llama (Lama glama) infectada con Sarcocystis aucheniae mediante métodos químicos: marinado, ahumado, curado seco y curado húmedo. Rev Inv Vet Perú 18: 57-63. 
9. Hiepe F, Lietzke L, Scheibner G, Jungmann R, Hiepe T, Montag T. 1981. Untersuchungen zur toxischen wirkung von extrankten aus Sarcocystis ovifelis-Macrocysten auf Kanichen. Mh Vet Med 36: 908-910.

10. Leguía G. 1991. The epidemiology and economic impact of llama parasites. Parasitol Today 7: 54-56.

11. Leguía G, Arévalo F. 1990. efecto de la cocción, refrigeración, congelación y deshidratación (charqui) sobre la viabilidad del Sarcocystis de alpacas. MV Rev Cienc Vet 6(1): 19-28.

12. Leguía G Casas E. 1999. Enfermedades parasitarias y atlas parasitológico de camélidos sudamericanos. Lima: Ed. De Mar. $190 \mathrm{p}$.

13. Leguía G, Guerrero C, Sam R, Chávez A. 1989. Infección experimental de perros y gatos con micro y macroquistes de Sarcocystis de alpacas (Lama pacos). MV Rev Cienc Vet 5(3): 10-13.

14. Leguía G, Guerrero C, Sam R, Rosadio R. 1990. Patología de Sarcocystis aucheniae en alpacas infectadas experimentalmente. MV Rev Cienc Vet 6(3): 11-13.

15. Mansilla D. 1993. Efecto histopatológico del lisado de macroquistes de Sarcocystis aucheniae en ratones, conejos y cobayos. Tesis de Médico Veterinario. Lima: Univ Nacional Mayor de San Marcos. 60 p.

16. Moreno M. 2003. Higiene e inspección de carnes 2. Madrid: Diaz de Santos. 593 p.

17. Mostajo W. 1983. Sarcocistiosis en alpacas beneficiadas en el camal municipal de Santa Rosa. Tesis de Médico Veterinario y Zootecnista. Puno: Univ Nacional del Altiplano. 68 p.
18. Nichpanit S, Nak W, Wongsaroj T, Nithikathkul C. 2010. First large scale of human Sarcocystis hominis in Thailand. Trends Res Sci Technol 2(1): 1-5.

19. Piekarski G, Heydorn AO, Aryeetey ME, Hartlapp JH, Kimmig P. 1978. Clinical, parasitoligical and serological investigations in sarcosporidosis (Sarcocystis suihominis) of man. Immun Infekt 6(4): 153-159.

20. Rojas M. 1990. Parasitismo de los rumiantes domésticos. Terapia, prevención y modelos para su aprendizaje. Lima: Ed Mijosa. 383 p.

21. Sam R, Mansilla I, Morales $C$, Ramírez A. 1998. Efecto tóxico de macroquistes de Sarcocystis aucheniae en ratones, cobayos y conejos. Rev Inv Pec IVITA 9(2): 11-18.

22. Sam R. 1988. Sarcocystis aucheniae: Caracterización parcial de componentes antigénicos y patología clínica experimental en alpacas. Tesis de Doctorado. Lima: Univ Nacional Mayor de San Marcos. $118 \mathrm{p}$.

23. Subercaseaux P. 1994. Sarcocystis spp. in human and domestic animals. Int J Parasitol 18: 821-827.

24. Vilca M. 1991. Producción, tecnología e higiene de la carne. En: Avances y perspectivas del conocimiento de los camélidos sudamericanos. Santiago de Chile: FAO. p 42-49.

25. Wilairatana P, Radomyos $\boldsymbol{P}$, Radomyos B, Phraevanich R, Plooksawasdi W, Chanthavanich P, et al. 1996. Intestinal sarcocystosis in the Thai laborers. Southe Asian J Trop Med Public Health 27(1): 43-46. 